\title{
The ROSAS Cohort: A Prospective, Longitudinal Study of Biomarkers for Alzheimer's Disease. Strategy, Methods and Initial Results
}

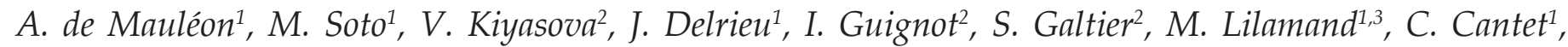 \\ F. Lala ${ }^{1}$, N. Sastre ${ }^{1}$, S. Andrieu ${ }^{1}$, M. Pueyo ${ }^{2}$, P.J. Ousset ${ }^{1}$, B. Vellas ${ }^{1}$
}

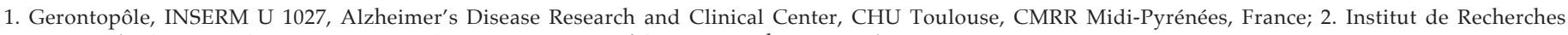
Internationales SERVIER, Suresnes, France; 3. APHP, Department of Geriatrics, Bichat Hospital, Paris, France

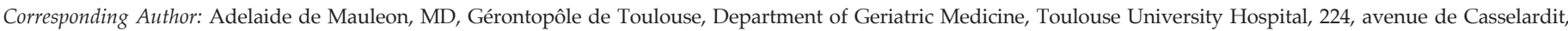
31059 TOULOUSE Cedex 9, France; Phone: 33.5.61.77.64.26, Fax: 33.5.61.77.64.78; E-mail: demauleon.a@chu-toulouse.fr

\section{Abstract}

OBJECTIVE: The aims of the Research Of biomarkers in Alzheimer's diseaSe (ROSAS) study were to determine the biofluid and imaging biomarkers permitting an early diagnosis of Alzheimer's disease and better characterisation of cognitive and behavioural course of the pathology. This paper outlines the overall strategy, methodology of the study, baseline characteristics of the population and first longitudinal results from the ROSAS cohort.

METHODS: Longitudinal prospective monocentric observational study performed at the Alzheimer's disease Research centre in Toulouse. A total of 387 patients were studied and analyzed in 3 groups: 184 patients with dementia of Alzheimer's type, 96 patients with memory disorders without dementia (Mild Cognitive Impairment) and 107 patients without abnormal memory tests (control group), and were followed up during 4 years. Patient's sociodemographic characteristics, risk factors, medical conditions, previous and current medications, neuropsychological assessment and overall cognitive status were recorded. Blood and urine samples were collected at every year, Magnetic Resonance Imaging were performed at inclusion, after one year of follow-up and at the end of the study.

RESULTS: At baseline, three different groups of the cohort differed interestingly in age, level of education, and in percentage of ApoEe4 carriers whereas the history of cardiovascular and endocrine pathologies were similar among the groups. During the follow-up period (3-4 years) 42 mild cognitive impairment patients $(43.8 \%)$ progressed to dementia, 7 controls progressed into mild cognitive impairment and 1 patient in the control group converted from mild cognitive impairment group to dementia of Alzheimer's type group. During the first year of follow up, the incidence of progression from mild cognitive impairment to dementia of Alzheimer's type was 12.7 per 100, during the second year 33.9 per 100 and 46.7 per 100 for the third year.

CONCLUSION: This paper presents the baseline characteristics of the unique French prospective monocenter study in which the natural course of dementia of Alzheimer's type was evaluated. Future analysis of blood and urine samples collection from the ROSAS study will permit to identify possible biofluid biomarkers predicting the early stages of the dementia of Alzheimer's type and risk of progression from Mild Cognitive Impairment to Alzheimer's disease.

Key words: Biofluid biomarkers, imaging biomarkers, Alzheimer's disease, early diagnosis.

\section{Introduction}

lzheimer's disease (AD) is the leading cause $\triangle$ of dementia, and because the primary risk 1 factor for AD is old age, the prevalence of the disease is increasing dramatically with ageing population worldwide. AD is a progressive, unremitting, neurodegenerative disorder that affects wide areas of the cerebral cortex and hippocampus. Abnormalities are usually first detected in the frontal and temporal lobes, and then slowly progress to other areas of the neocortex at rates that vary between individuals (1). The natural history of $\mathrm{AD}$ is characterized by a long preclinical stage that begins years or maybe decades prior to the onset of clinical symptoms (2).

The diagnosis of $\mathrm{AD}$ in clinical care is made however at the symptomatic - dementia stage. Overlapping but slightly different sets of criteria for dementia were provided by the International Classification of Diseases (ICD-10), used in Europe, and the US Diagnostic and statistical Manual of Mental Disorders (DSM - 5). These criteria list clinical features, necessary to make the clinical diagnosis of typical AD, and acknowledge atypical presentations as well. However, several studies demonstrated that people with the clinical diagnosis of $\mathrm{AD}$, when followed to postmortem, do not always have AD-type pathology, with around $20 \%$ suggested to be misclassified (3). Making a diagnosis of Alzheimer's Disease on purely clinical grounds is thus very challenging. New robust and affordable methods are needed for the exact diagnosis of the disease at its early stages and validation of dementia progression.

In the past years, several accurate AD biomarkers that can help the disease diagnosis have emerged (4). These are amyloid $\beta 1-42$, total tau and phosphorylated tau levels measured in the cerebrospinal fluid (CSF)(5). Functional and molecular imaging technologies have 
confirmed that brain amyloid deposition begins decades before dementia (in asymptomatic subjects) and precedes cognitive decline and brain atrophy (6). The complete biomarker profile may thus help to identify individuals at early stages, who may draw substantial benefit from disease-modifying therapy or intervention, aiming to change the course of the disease from the preclinical stage.

Not only clinical but also potential economic benefits of early AD diagnosis have been extensively described (7). The sooner a patient is diagnosed with AD, the sooner an appropriate management and targeted interventions might be offered and might thus permit to decrease the economic impact. However, the current biomarkers present some limitations restricting their implementation in clinical routine. Invasive collection methods (e.g. lumbar puncture), additional costs, limited access to neuroimaging centers and the absence of reliable validated standardized blood biomarkers raise major issues. Additional efforts are still needed for identifying reliable, easily exploitable and sensitive circulating biomarkers of AD pathophysiology (e.g. metabolic and inflammatory markers, intra- and extracellular proteins, hormones, miRNA) that will provide highly specific diagnosis of the disease.

The aim of the Research Of biomarkerS in Alzheimer's diseaSe (ROSAS) study is to determine new biofluid and imaging biomarkers that are associated with earlier diagnosis of $\mathrm{AD}$ and better characterising of the cognitive and behavioural course of the disease, in 3 groups: patients with dementia due to $\mathrm{AD}(\mathrm{AD})$, patients with memory disorders without dementia (Mild Cognitive Impairment (MCI)) and participants with a normal neuropsychological performance (control group). This paper outlines the overall strategy, methodology of the study, baseline characteristics of the population and first results of their longitudinal evolution longitudinal.

\section{Methods}

\section{Design}

The prospective ROSAS cohort is a longitudinal monocentric observational study performed at the ADResearch centre in Toulouse, France. The population was comprised of 3 groups of subjects:

(a) patients with dementia of Alzheimer's Type (mild to moderate stage),

(b) patients with memory disorders without dementia (MCI),

(c) subjects with normal neuropsychological performance (control group). Clinical visits were scheduled at baseline and twice a year for AD and MCI patients and once a year for control participants, during a 4-year follow up period (Table 1). Participants were included between July 2007 and March 2011. The last follow up visit took place in March 2014.

\section{Population}

Study population was recruited in a memory clinic setting of Toulouse University Hospital. Participants were men and women, aged 65 years and older, enrolled in 3 following groups:

(a) Patients with Alzheimer's dementia met Alzheimer's dementia criteria of Diagnostic and Statistical Manual version IV revised (DSM-IV-TR)(8), had a Mini-Mental State Examination (MMSE)(9) score of 12-26 and global Clinical Dementia Rating Scale (CDR) (10) score of 0.5 or higher (mild to moderate stage); (b) $\mathrm{MCI}$ patients did not meet diagnostic criteria of dementia due to AD of DSM-IV-RT, had memory disorder detected by the Rey Auditory Verbal Learning test (RAVLT)(11) $(<1$ standard deviation (SD) of the age-adjusted mean), a MMSE score of 24 or higher and a CDR global score of 0.5 ;

(c) Control subjects could have or not memory complaint at the interview and had no memory impairment detected by the RAVLT (value within \pm 1 SD of the age-adjusted mean), had a MMSE score of 26 and higher and a CDR global score of 0 .

Participants unable to speak or write French, under legal protection, subjects with brain tumor, stroke or other neurologic diseases that may explain cognitive deficit (e.g. Parkinson's disease, multiple sclerosis, epilepsy...), with a diagnoses of vascular dementia according to the NINDS-AIREN criteria (12), or other types of dementia, with serious illness or participating in a clinical trial were excluded.

Participants and their informal caregiver took part in the study on voluntary basis, and, they gave their written informed consent at selection. Ethics committee of Toulouse University Hospital approved the study protocol and all its amendments.

\section{Data collection}

At baseline and at every follow-up visit face-to-face interviews were held for neuropsychological evaluation of study participant by trained neurologist. Table 1 shows the investigation schedule according to the cognitive status of participants.

Clinical examination of study participants, recording of on-going treatments and reporting of adverse events were done twice a year for patients with dementia due to $\mathrm{AD}$ and $\mathrm{MCI}$ and once a year for control subjects. Neuropsychological assessments and records of cognitive decline progression (control to $\mathrm{MCI}$ and/or $\mathrm{AD}, \mathrm{MCI}$ to $\mathrm{AD}$ ) were conducted by trained neuropsychologists at the same periodicity for three different groups.

Brain Magnetic Resonance Imagings (MRIs) were performed on optional basis at enrolment, and then at 12 months and 4-year time points using 3-Tesla scan and included the acquisition of several different sequences: 3 dimensional T1 and T2; 2-dimensional Axial Fluid 
Attenuated Inversion Recovery (FLAIR), axial Diffusion Weighted Imaging (DWI), axial Diffusion-Tensor Imaging (DTI); and axial Susceptibility Weighted Imaging (SWI).

Biological samples (blood and urine) were collected in this study at the following timepoints: the first visit (month 0-M0) and at M12, M24, M36 and M48. Samples were stored in the Cellular Biology and Cytology Laboratory of Toulouse University Hospital and later transferred to specialized CRO B\&C (Mechelen, Belgium). The blood cell samples were used for the analysis of the cellular proteins and RNA extraction. The DNA extracted from the whole blood sample at baseline was used for participants' APOE genotyping. Other genes are planned to be analysed further.

Compulsory lumbar punctures were not a part of the protocol. However, if a lumbar puncture was performed before the participants' enrolment into ROSAS cohort or it was part of his/her clinical follow-up, $1 \mathrm{ml}$ of CSF could have been used for the purposes of this study with the participant's consent.

\section{Demographics}

Subjects' level of education, age, gender and living arrangement were collected using a structured questionnaire directed to patient and/or their caregivers.

\section{Medical characteristics}

Medical history of past and current clinical conditions was recorded with focus on risk factors, cardiac, vascular and psychiatric diseases. At each clinical visit, a neurological examination was performed, height and weight were measured and body mass index was calculated.

Pharmacological treatments with focus on psychotropic medications (anxiolytic, antipsychotic, sedative, antidepressant drugs) as well as cardiac treatment (renin-angiotensin system inhibitors, beta blockers, calcic inhibitors and diuretics) were recorded by a structured questionnaire.

As for anti-dementia treatments, specific drugs including memantine and acetylcholinesterase inhibitors were recorded separately from other non- specific treatments: herbal mixtures, vitamins, traditional medicine.

\section{Clinical assessment}

\section{Cognitive assessment included}

- MMSE to evaluate orientation, memory, attention, concentration, denomination, repetition, comprehension, ability to formulate a whole sentence and to copy polygons. The disease severity was defined as mild (MMSE $>20)$ and moderate (12-20). Patients at severe stage
(MMSE<12) were excluded.

- Global CDR score in order to provide a global rating of severity of dementia on a scale ranging from 0 (no impairment) to 3 (severe impairment). Ratings were taken for 3 cognitive domains (memory, orientation, judgment and problem solving) and 3 functional domains (community affairs, home and hobbies, personal care.). All patients included with a diagnosis of AD had a CDR of 0.5 or above. The severity of disease was defined according to global score as none $(\mathrm{CDR}=0)$, questionable $(\mathrm{CDR}=0.5)$, mild $(\mathrm{CDR}=1)$, moderate $(\mathrm{CDR}=2)$ and severe $(C D R=3)$. Patients at severe stage $(C D R=3)$ were excluded. The CDR sum of boxes (CDR SOB) represents the addition of all scores of cognitive and functional domains.

- Alzheimer Disease Assessment Scale (ADAS-Cog 11 items)(13), to assess various cognitive functions: language, comprehension, denomination, orientation, memory and execution of orders. The total score ranges from 0 to 70 , with higher scores indicating the more severe cognitive impairment. This scale was administered to patients with dementia due to $\mathrm{AD}$ and those with MCI.

- RAVLT to evaluate the explicit verbal episodic memory via learning and recall of sum of words. This test was administered only to control subjects and patients with MCI.

- Trail Making Test (TMT) A and B (14) to judge perceptive-cognitive-motor speed in part $\mathrm{A}$ and mental flexibility capacities in part B. This test was administered only to control subjects and patients with MCI.

- Time of onset and duration (in months) of cognitive impairment and memory complaints in $\mathrm{AD}$ and $\mathrm{MCI}$ subjects.

- Worsening of cognitive status according to the baseline status of participants: conversion of controls to $\mathrm{MCI}$, conversion of controls to $\mathrm{AD}$ and conversion of $\mathrm{MCI}$ to $\mathrm{AD}$. In this study, the definition of conversion included changes in psychometric scores (inclusion criteria for each group) combined with clinical judgment of neuropsychiatrist having evaluated the patient. If one of the total scores (MMSE or CDR) were not exactly as defined per protocol for the particular group - final decision on conversion or not was taken by the MD.

\section{Functional evaluation was performed by administration of}

- Physical impairment based on Alzheimer's Disease Cooperative Study-Activities of Daily Living (ADCSMCI-ADL) scale (15). This is a 24-item scale to measure daily living activities. Total score ranges from 0 to 78 . A higher score indicates less functional impairment.

Neuropsychiatric symptoms (NPS) were measured by total and individual items of the neuropsychiatric inventory (NPI-12) scale (16). The score ranges from 0 to 144, with higher scores indicating the presence of more (severe) NPS. 


\begin{tabular}{|c|c|c|c|c|c|c|c|c|c|}
\hline Assessments & BL & $\begin{array}{l}\text { Follow- } \\
\text { up M6* }\end{array}$ & $\begin{array}{l}\text { Follow- } \\
\text { up M12 }\end{array}$ & $\begin{array}{l}\text { Follow- } \\
\text { up M18* }\end{array}$ & $\begin{array}{l}\text { Follow- } \\
\text { up M24 }\end{array}$ & $\begin{array}{c}\text { Follow-up } \\
\text { M30* }\end{array}$ & $\begin{array}{l}\text { Follow-up } \\
\text { M36 }\end{array}$ & $\begin{array}{l}\text { Follow-up } \\
\text { M42* }\end{array}$ & $\begin{array}{c}\text { End of } \\
\text { study M48 }\end{array}$ \\
\hline Informed consent & $X$ & & & & & & & & \\
\hline Inclusion criteria & $\mathrm{X}$ & & & & & & & & \\
\hline DSM-IV-TR AD criteria & $X$ & & & & & & & & \\
\hline Medical history & $X$ & & & & & & & & \\
\hline Clinical examination & $X$ & $x$ & $x$ & $x$ & $x$ & $x$ & $x$ & $x$ & $x$ \\
\hline Ongoing treatments & $X$ & $x$ & $x$ & $x$ & $x$ & $x$ & $x$ & $x$ & $x$ \\
\hline MRI (optional) & $X$ & & $x$ & & & & & & $x$ \\
\hline Rey Auditory Verbal Learning & $X$ & $X$ & $x$ & $x$ & $X$ & $X$ & $x$ & $x$ & $x$ \\
\hline CDR & $X$ & $x$ & $x$ & $x$ & $x$ & $x$ & $x$ & $x$ & $x$ \\
\hline MMSE & $X$ & $X$ & $x$ & $X$ & $x$ & $x$ & $x$ & $x$ & $x$ \\
\hline $\mathrm{NPI}^{*}$ & $X$ & $x$ & $x$ & $x$ & $x$ & $x$ & $x$ & $x$ & $x$ \\
\hline ADCS-MCI-ADLI* & $X$ & $x$ & $x$ & $x$ & $x$ & $x$ & $x$ & $x$ & $x$ \\
\hline ADAS-Cog* & $X$ & $x$ & $x$ & $x$ & $x$ & $x$ & $x$ & $x$ & $x$ \\
\hline TMT A and B & $X$ & $x$ & $x$ & $X$ & $x$ & $X$ & $x$ & $x$ & $x$ \\
\hline Conversion to $\mathrm{AD}^{*}$ & & $X$ & $x$ & $x$ & $x$ & $x$ & $x$ & $x$ & $x$ \\
\hline \multicolumn{10}{|l|}{ General Follow-up criteria } \\
\hline Biological tests & $x$ & & $x$ & & $x$ & & $x$ & & $x$ \\
\hline Adverse events & & $x$ & $x$ & $X$ & $x$ & $x$ & $x$ & $x$ & $x$ \\
\hline \multicolumn{10}{|l|}{ Samples** } \\
\hline Blood sample: DNA, & $x$ & & & & & & & & \\
\hline Plasma/serum and blood cells & $x$ & & $x$ & & $\mathrm{X}$ & & $x$ & & $x$ \\
\hline Urine collected & $x$ & & $x$ & & $x$ & & $x$ & & $x$ \\
\hline
\end{tabular}

${ }^{*}$ Only for patients with demonstrated memory disorders or Alzheimer's disease; ** Sample of cerebrospinal fluid is performed only if a lumbar puncture is indicated as part of the patient's follow-up outside the study; ${ }^{\circ}$ Memory complaint and cognitive impairment only for AD and MCI subjects.

\section{Laboratory test assessment}

\section{Blood}

At baseline, complete blood cells count, proteinaemia, albuminaemia, creatininaemia; total cholesterol, triglycerides, C Reactive Protein (CRP), vitamin B12, folates and homocysteine were evaluated in blood samples. Each year, laboratory test assessment included complete blood cells count, proteinaemia, albuminaemia, creatininaemia, total cholesterol, triglycerides and CRP.

In addition, a part of sample collection was oriented for research purpose. Proteomic, metabolomic and transcriptomic analyses were performed in order to identify new potential biomarkers, and to compare protein, metabolic and RNA profiles of different groups of patients.

\section{Urine}

Urine strip test was performed at inclusion and urine samples collected once a year were destined for research purposes.

\section{CSF}

Total tau protein, phosphorylated tau protein and amyloid protein $(\mathrm{A} \beta 42)$ were analysed in CSF using ELISA (Innotest hTAU-Ag and Innotest b-amyloid (1-42), Innogenetics) for clinical diagnosis confirmation. Remaining CSF was used for the research of new markers qualified of unidentified markers.

\section{Genetic data}

ApoEع4 genotyping was performed for all participants and defined the presence of one or both alleles (" carrier" status) or the absence of ApoE\&4 allele ("non-carrier status").

\section{Statistical analysis}

Descriptive statistics were provided by participants group. For quantitative data, the mean and standard deviation (SD) were presented. For qualitative data, number of observed values, number and percentage of participants were presented. All analyses were performed 


\begin{tabular}{|c|c|c|c|c|}
\hline & $\begin{array}{c}\text { Control population } \\
\mathbf{n}=107(27.6 \%)\end{array}$ & $\begin{array}{c}\text { MCI population } \\
\mathrm{n}=96(24.8 \%)\end{array}$ & $\begin{array}{c}\text { AD population } \\
\mathrm{n}=184(47.5 \%)\end{array}$ & $\mathrm{p}$ \\
\hline Age, years* & $75.6 \pm 6.0$ & $78.7 \pm 5.6$ & $79.5 \pm 5.6$ & $<0.001$ \\
\hline \multicolumn{5}{|l|}{ Age, n (\%): } \\
\hline$-80-90$ & 32 & 43 & 92 & - \\
\hline$->90$ & 0 & 1 & 4 & - \\
\hline Female, n (\%) & $53(49.5 \%)$ & $57(59.4 \%)$ & $124(67.4 \%)$ & 0.01 \\
\hline Education, number of years* & $9.7 \pm 4.2$ & $8.4 \pm 3.9$ & $7.6 \pm 3.5$ & $<0.001$ \\
\hline Tobacco $^{\circ}$ & $44(41.1 \%)$ & $30(30.3 \%)$ & $40(21.7 \%)$ & - \\
\hline Hypercholesterolemia & $46(41.8 \%)$ & $49(49.5 \%)$ & $70(35.2 \%)$ & 0.83 \\
\hline $\mathrm{BMI}^{*}\left(\mathrm{~kg} / \mathrm{m}^{2}\right)$ & $26.2 \pm 3.9$ & $26.8 \pm 4.3$ & $25.8 \pm 3.96$ & 0.16 \\
\hline \multicolumn{5}{|l|}{ Genetics } \\
\hline ApoE4, total n $(\%)$ & $22(20.6 \%)$ & $34(35.4 \%)$ & $88(48.1 \%)$ & $<0.001$ \\
\hline \multicolumn{5}{|l|}{ ApoE4, status n (\%): } \\
\hline - Homozygous & 0 & $8(8.3 \%)$ & $13(7.1 \%)$ & - \\
\hline - Heterozygous & $22(20.6 \%)$ & $26(27.1 \%)$ & $75(41.0 \%)$ & - \\
\hline
\end{tabular}

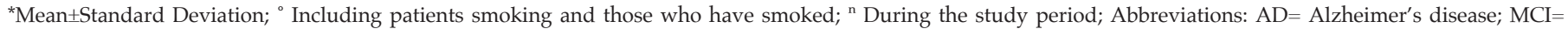
Mild Cognitive Impairment; BMI=Body Mass Index; ARAS=Agent acting on the rennin-angiotensin system.

on observed values, missing data were not imputed. Comparisons between groups were performed, using Chi2 statistics (or Fisher exact test for expected values $<5)$ for discrete outcome, and using a General Linear Model for continuous outcomes. Time to conversion was analyzed by Kaplan-Meier method to estimate the percentage of converted participants with $95 \%$ confidence interval. Moreover, subgroups analysis was performed, defined according to the phenotype of participants at enrolment (control, MCI, AD; severity of AD; age class; gender; ApoEع4), and their status of conversion or not during the study.

Data were analyzed with SAS software.

\section{Results}

408 participants were included at baseline visit: 110 controls, $99 \mathrm{MCI}$ and $199 \mathrm{AD}$ patients. Among the total population, twenty-one subjects were excluded from the analysis because of the protocol deviations: age (<65 years), neurological disease (epilepsy, Parkinson's disease) and MMSE $<12$. The current results concern 387 participants who completed their baseline visit: 107 controls, $96 \mathrm{MCI}$ and $184 \mathrm{AD}$ (Figure 1). For all the analyses described in this article participants were divided into groups according to their baseline status. At the end of the study, 78 participants in the control group, 64 patients in the MCI group and 107 $\mathrm{AD}$ patients completed the 4-year follow up visit. One hundred thirty-eight study participants (35.7\%) dropped out of the study for non-medical and medical reasons. At baseline, two thirds of the population were female. The mean age was $79.5 \pm 6.0$ for patients with dementia due to $\mathrm{AD}, 78.7 \pm 5.6$ for MCI patients and $75.6 \pm 6.0$ for controls $(p<0.001)$. Almost half of the patients with AD, one-third of MCI subjects and one-fifth of controls were ApoE $\varepsilon 4$ carriers $(\mathrm{p}<0.001)$. Table 2 shows the baseline socio demographic characteristics, known risk factors (cardiovascular included) of the population of the ROSAS study, comparing the 3 groups. Major risk factors showed no statistically significant difference among groups.

As for the medical history (Table 3), the main difference was observed in psychiatric diseases - AD patients presented more often history of depression than controls $(p=0.01)$. In the panel of blood analysis, the only slight difference was observed in homocysteine concentrations - elevated in AD vs. MCI vs. controls $(p<0.001)$. The pharmacological treatments, prescribed to the participants before and during the study are listed in Table 3. The psychotropic treatments were prescribed most commonly in the patients with $\mathrm{AD}$ than in controls $(p<0.001)$ whereas the prescription of cardio- vascular medications was similar in different groups. It is important to note that data on anti-dementia 


\begin{tabular}{|c|c|c|c|c|}
\hline & $\begin{array}{l}\text { Control population } \\
n=107(27.6 \%)\end{array}$ & $\begin{array}{c}\text { MCI population } \\
n=96(24.8 \%)\end{array}$ & $\begin{array}{l}\text { AD population } n=184 \\
(47.5 \%)\end{array}$ & $\mathrm{p}$ \\
\hline \multicolumn{5}{|l|}{ Vascular diseases, $\mathrm{n}(\%)$} \\
\hline Ischemic and hemorrhagic stroke & $2(1.9 \%)$ & $1(1.0 \%)$ & $7(3.8 \%)$ & 0.33 \\
\hline \multicolumn{5}{|l|}{ Endocrine diseases, n (\%) } \\
\hline \multicolumn{5}{|l|}{ Psychiatric, n (\%) } \\
\hline Depression & $23(21.5 \%)$ & $36(37.5 \%)$ & $68(36.9 \%)$ & 0.01 \\
\hline Anxiety & $5(4.7 \%)$ & $6(6.3 \%)$ & $5(2.7 \%)$ & 0.35 \\
\hline Sleep disorders & $12(11.2 \%)$ & $10(10.4 \%)$ & $16(8.7 \%)$ & 0.77 \\
\hline - Anxiolytic & $12(10.9 \%)$ & $27(27.3 \%)$ & $84(42.2 \%)$ & - \\
\hline - Antipsychotic & - & $3(3.0 \%)$ & $35(17.6 \%)$ & - \\
\hline - Sedative & $15(13.6 \%)$ & $18(18.2 \%)$ & $38(19.1 \%)$ & - \\
\hline - Antidepressant & $22(20.0 \%)$ & $43(43.4 \%)$ & $102(51.3 \%)$ & - \\
\hline \multicolumn{5}{|l|}{ Antidementia $^{\mathrm{n}}$ : } \\
\hline - Total & $23(21.5 \%)$ & $47(49.5 \%)$ & $178(97.3 \%)$ & $<0.001$ \\
\hline - Memantine & $0(0.0 \%)$ & $14(15.2 \%)$ & $119(64.8 \%)$ & - \\
\hline - Anticholinesterases & $3(2.7 \%)$ & $37(39.4 \%)$ & $167(91.5 \%)$ & - \\
\hline - Diuretics & $16(14.5 \%)$ & $21(21.2 \%)$ & $37(18.6 \%)$ & - \\
\hline
\end{tabular}

${ }^{*}$ Mean \pm Standard Deviation; ${ }^{\mathrm{n}}$ During the study period; Abbreviations: $\mathrm{AD}=$ Alzheimer's disease; $\mathrm{MCI}=\mathrm{Mild}$ Cognitive Impairment; ARAS=Agent acting on the rennin-angiotensin system.

treatments was reported for both: specific and non specific treatments ("others" - multivitamins, ginkgo biloba, etc.). As mentioned earlier, baseline status of study participants was taken into account for this analysis, however, some of them progressed during the study duration to MCI or to AD. Thus, 3 control participants using acetylcholinesterase inhibitors are those who progressed to $\mathrm{MCI}$ and to $\mathrm{AD}$. As for $18.7 \%$ of control subjects to whom "other anti-dementia drugs" were prescribed, these are participants who have been receiving mostly Gingko preparations for dementia prevention.

Table 4 highlights the results of cognitive, functional and neuropsychological assessment of ROSAS study participants at baseline. As expected, the cognitive impairment was significantly more severe in $A D$ population than in MCI subjects or controls $(\mathrm{p}<0.001)$. As for the functional and behavioral impairment, the patients with dementia were significantly more functionally $(p<0.001)$ and behaviorally $(p<0.001)$ disabled than $\mathrm{MCI}$ and control participants. Interestingly, when baseline cognitive scores were compared among ApoE 44 carriers and non-carriers trends to difference were observed in all three groups including controls despite the small sample size (Supplementary Table 1).

During the 4-year follow up, $42 \mathrm{MCI}$ patients converted to dementia, 5 controls progressed into MCI group and 4 control subjects evolved to AD group. Figure 2 describes the time to conversion from patients with $\mathrm{MCI}$ to AD status. During the first year follow up, the incidence of progression from MCI to AD was $12.7 \%$ (95\% Confidence Interval $(\mathrm{CI})=7.3 \%-21.8 \%)$, during the second year $33.9 \%(95 \% \mathrm{CI}=24.7 \%-45.2 \%)$ and $46.7 \%$ $(95 \% \mathrm{CI}=36.2 \%-58.4 \%)$ in the third year respectively. The incidence rate of conversions from $\mathrm{MCI}$ to $\mathrm{AD}$ status on the M0-M12 period was 12.9 events for 100 patient-years, 


\begin{tabular}{|c|c|c|c|c|}
\hline & $\begin{array}{l}\text { Control population } \\
n=107(27.6 \%)\end{array}$ & $\begin{array}{c}\text { MCI population } \\
n=96(24.8 \%)\end{array}$ & $\begin{array}{c}\text { AD population } \\
n=184(47.5 \%)\end{array}$ & p \\
\hline MMSE, total* & $29.1 \pm 1.2$ & $26.2 \pm 2.0$ & $19.8 \pm 4.1$ & $<0.001$ \\
\hline \multicolumn{5}{|l|}{ MMSE, stage n $(\%)$ : } \\
\hline ADAS-Cog, total ${ }^{*}$ & - & $8.4 \pm 2.5$ & $17.5 \pm 7.7$ & $<0.001$ \\
\hline Sum of boxes CDR score* & $0.01 \pm 0.07$ & $1.20 \pm 0.75$ & $6.02 \pm 2.74$ & $<0.001$ \\
\hline \multicolumn{5}{|l|}{ CDR, stage n $(\%)$ : } \\
\hline - CDR 0 & $106(99.1 \%)$ & $2(2.1 \%)$ & $0(0.0 \%)$ & \\
\hline TMT A, total (seconds)* & $40.5 \pm 14.5$ & $69.0 \pm 25.1$ & $109.5 \pm 59.9$ & $<0.001$ \\
\hline TMT B, total (seconds) ${ }^{*}$ & $97.8 \pm 40.0$ & $187.3 \pm 74.3$ & $203.8 \pm 111.1$ & $<0.001$ \\
\hline \multicolumn{5}{|l|}{ Autonomy } \\
\hline ADCS-MCI-ADL total score* & - & $43.9 \pm 6.0$ & $29.1 \pm 10.9$ & $<0.001$ \\
\hline \multicolumn{5}{|l|}{ Behavioral disorders } \\
\hline NPI, total ${ }^{*}$ & - & $9.5 \pm 10.1$ & $16.9 \pm 16.4$ & $<0.001$ \\
\hline
\end{tabular}

*Mean \pm Standard Deviation; Abbreviations: $\mathrm{AD}=$ Alzheimer's disease; NPI= NeuroPsychiatric Inventory-Questionnaire; MCI= Mild Cognitive Impairment; MMSE=Mini Mental State Examination; CDR= Clinical Dementia Rating scale; ADAS-cog=Alzheimer Disease Assessment Scale; TMT= Trail Making Test; ADCS-MCIADLI=Alzheimer's Disease Cooperative Study-Activities of Daily Living Prevention.

19.5 events for 100 patient-years on M0-M24 period, 20 events for 100 patient-years on M0-M36 period and on M0-M48 period, 20.1 events for 100 patient-years.

Since lumbar punctures were not compulsory for this study, only 21 CSF samples requested by clinician for diagnosis confirmation had been obtained and analysed. One lumbar puncture was performed before the participant's enrolment into the study, whereas others were performed during 4-year follow up: 11 AD patients, $8 \mathrm{MCI}$ and 1 normal control (Supplementary Table 2). In 5 cases from 21, biological evaluation helped to confirm the diagnosis of $\mathrm{AD}$. When the CSF profile was unhelpful, the final diagnosis relied on clinical judgment.

\section{Discussion}

During the past two decades, the steady incremental progress was being made in understanding the natural history of Alzheimer's disease, the kinetics of the disease evolution, its interaction with co-morbidities and normal brain ageing. Epidemiological and genetic studies have identified many risk factors that increase the risk of AD. Prevention studies have highlighted the possibility of targeting both risks and protective factors to delay the onset of the dementia. Several longitudinal cohorts providing better understanding of the disease naturalistic course and aiming the identification of biomarkers for its early diagnosis were launched in the North America (ADNI) and Australia (AIBL). However, taking into account notable differences in ethnic, cultural, socioeconomic factors and life style between USA and Europe, the results of such studies should be extrapolated on European population with caution. Specific longitudinal European cohorts are thus of a particular need.

\section{ROSAS baseline}

To our knowledge, the ROSAS cohort is the first and unique French monocenter study (2007 - 2014) that aimed better understanding of the course of the disease and creation of a biobank of samples from control subjects, MCI and dementia patients for further biomarkers' research. The findings of this study demonstrate a certain number of similarities when baseline characteristics of the ROSAS cohort population are compared with that of ADNI (or AIBL). However, the important differences were observed in the level of education, percentage of ApoE 44 carriers and concomitant medications per group between ROSAS and ADNI.

From the point of view of natural disease progression, the main finding of the ROSAS cohort was that almost half of the patients with MCI $(n=42(43.8 \%))$ converted to dementia during the 4-year follow-up period, whereas in control group only 9 subjects $(8.4 \%)$ declined cognitively 
during the same period converting to $\mathrm{MCI}$, to $\mathrm{MCI}$ and $\mathrm{AD}$ or to $\mathrm{AD}$ directly.

The summary of the baseline characteristics of the subjects indicated significant differences in sociodemographic characteristics among groups, including age, gender and the number of years of former education. The AD patients were the oldest and with the lowest level of education. These observations are in accordance with some baseline data from ADNI (mean age and gender). As for the level of formal education the population enrolled in the ROSAS cohort seems closer to data from ICTUS study and corresponds to "real-life" elderly subjects from Western European countries (14.7 years of education in ADNI vs. 7.6 in ROSAS vs. 9.7 in ICTUS) (17-19).

\section{Risk factors}

Dementia is a multifactorial disorder determined by the interplay of the genetic susceptibility and different risk factors, among which cardiovascular risk factors (hypertension, diabetes, obesity, etc.) and smoking (even second hand smoking) were shown to be associated with $50 \%$ increased risk of dementia (20). In the ROSAS cohort, no statistically significant differences were identified in vascular and metabolic factors. Main cardiovascular pathologies as well as endocrine diseases were comparable among phenotypes. Pharmacological treatment for these pathologies was similar for 3 groups.

However, an interesting parameter that showed significant difference was the concentration of homocysteine in blood. Elevated homocysteine levels have been associated with an increased risk of cognitive impairment and dementia. However, the capacity of this parameter alone or in combination with other factors to predict the cognitive decline is being still actively debated. Kim et al, have analyzed the relationship of cognitive function with homocysteine, vitamin B and tissue factor pathway inhibitor in cognitively impaired elderly in a cross-sectional survey and demonstrated that plasma homocysteine levels were higher in patients with $\mathrm{AD}$ and $\mathrm{MCI}$ than those in normal subjects and were negatively correlated with Word List Memory and Constructional Recall Test (21). Another study, performed in Europe - in a community dwelling cohort of older adults from the Vienna Transdanube aging study with MCI showed that hyperhomocysteinaemia at baseline was a predictor of moderate to severe brain atrophy in these subjects in five years (22). As for the results from AIBL - homocysteine levels were increased in female AD patients compared to female Healthy Control subjects (p-value $<0.001$ ), but not in males. However composite z-scores of short- and long-term episodic memory, total episodic memory, and global cognition showed significant negative correlations with homocysteine, in all clinical categories, underscoring the association of this parameter with cognitive deterioration (23).
The specificity of this parameter alone is still being discussed, since homocysteine imbalance has long been linked to cognitive dysfunction in schizophrenia for example (24). However, recent publication from Doody et al. demonstrated that elevated homocysteine was associated with $\mathrm{AD}$, suggesting that it might promote the accumulation of the DNA damage in neurons and sensitize them to amyloid beta protein toxicity (25). We can thus suggest that in further multi-modal analyses the combination of homocysteine concentration in blood with specific MRI parameters standardized for neurodegenerative diseases and cognitive scales scores at baseline in the ROSAS cohort patients may be explored as a combined predictive factor of conversion from $\mathrm{MCI}$ to AD.

Recent intensive genetic research has identified Apo$\varepsilon 4$ as a susceptibility gene for sporadic AD. But only $50 \%$ of the late-onset $\mathrm{AD}$ subjects were shown to be Apo- $\varepsilon 4$ carriers and thus, it is not currently used alone as a biomarker of $\mathrm{AD}$ diagnosis and progression. It is known that humans expressing ApoE4 protein develop more plaques and vascular $\beta$-amyloid deposits than those expressing only ApoE3 (26) and this has been confirmed in genetically engineered mice (27). In the ROSAS study, almost half of patients with dementia of AD type were Apo- $\varepsilon 4-$ carriers $(48 \%)$, whereas in ADNI and AIBL this percentage was slightly higher for both AD patients $(66 \%$ and $61 \%)$ and healthy controls (26\% and $27 \%$ ) respectively with a higher sample size as well (28). It is to note that when carriers and non-carriers were compared in each subgroup (control vs. MCI vs. $\mathrm{AD}$ ) and the differences in cognitive performances evaluated by several scales were documented already at baseline. The data from AIBL study demonstrated same slight variations in baseline cognitive performances among subjects with different genotype. Moreover, the proportion of APOE $\varepsilon 4$ carriers differed between stable MCI stable (36.9\% APOE $\varepsilon 4$ positive) and MCI patients who progressed to $\mathrm{AD}(78.1 \% \mathrm{APOE} \varepsilon 4$ positive) after 18 months of follow-up (29) indicating its important impact on increasing the risk of cognitive decline progression. Further analysis of several genes that might be potential risk factors for $\mathrm{AD}$ and conversion from $\mathrm{MCI}$ to $\mathrm{AD}$, including triggering receptor expressed on myeloid cells 2 (TREM2), cluster of differentiation 33 (CD33), clusterin (CLU), complement receptor 1 (CR1), phosphatidylinositol binding clathrin assembly protein (PICALM), and sortilin-related receptor (SORL1) will be performed shortly.

\section{Treatments}

In the present cohort, we found high rates of specific anti-dementia treatment including memantine, anticholinesterases drugs. Almost $100 \%$ of patients with $\mathrm{AD}$ dementia and a half of patients with MCI had a treatment. Interestingly in north American cohorts as 
ADNI (30) or Canadian Outcomes Study in Dementia (COSID) (18), the percentage of AD subjects using these therapies at baseline is slightly lower and is around $85 \%$. A possible reason for higher prevalence of specific treatment in our cohort is that it is quite recent; subjects have been identified relying on multiplicity of specific criteria, evaluated by experienced neuropsychologists in a center for memory disorders and AD research, which does not reflect the real clinical practice. Moreover, it could be hypothesized that anti-dementia use would be variable because of national dementia policies and the existing guidelines. For example, a recent European cohort with $\mathrm{AD}$ subjects shows instead a higher rate of use of anti-dementia medication than in USA of 90\% (31).

The fact that can seem surprising is the level of prescription of nonspecific anti-dementia treatment found in our normal group. One-fifth on the control subjects were either, on vitamin $\mathrm{E}$ or gingko biloba, since our $\mathrm{AD}$ research center was participating in GUIDAGE study at the moment and participants who terminated the trial had the right to be enrolled in the ROSAS cohort. Another possible reason of the higher prevalence of treatment in the control population was the analysis bias - the definition of groups was done according to the baseline status of participants without taking account the change of cognitive status (e.g. conversion of $\mathrm{MCI}$ to $\mathrm{AD}$ group), thus suggesting the possibility that 3 control subjects receiving anticholinesterase drugs were those from 9 progressors.

However, when the prescription of psychotropic drugs was analyzed, we observed that they were prescribed most commonly in patients with AD. Previous studies in Europe (31) showed similar results and demonstrated that the prevalence of psychotropic treatment increases with the AD and with the stage of dementia. In ROSAS cohort antidepressants prescription was only slightly different between MCI and AD patients (43\% vs. 51\%), whereas the use antipsychotics was increased in AD patients. $18 \%$ of $\mathrm{AD}$ patients received antipsychotics vs. $3 \%$ of MCI participants. These drugs are more likely to be used in clinically severe patients and might be prescribed for various reasons, including hallucination, delusion, aggression, agitation, irritability, and sleep disturbances. The similar use of antidepressants in both groups might suggest that depression neither increased nor decreased over time but persisted as stable symptom present independently of cognitive decline progression. Finally, the different groups did not differ in terms of cerebrovascular or cardiovascular diseases or cardiac treatment.

\section{Longitudinal conversion}

During the 1st year of follow up, the conversion from $\mathrm{MCI}$ to AD status showed an incidence rate of 12.9 events from patient-years $(12.7 \%$ (95\% Confidence Interval (CI) $=7.3 \% ; 21.8 \%$ ). while on M0-M24 period the incidence increased to 19.5 events for 100 patient-years (33.9\%
(95\% Confidence Interval $(\mathrm{CI})=24.7 \% ; 45.2 \%)$, when 4-year period (M0-M48) was analyzed, the incidence of conversions in the ROSAS cohort was estimated as 20.1 events for 100 patient-years. In ADNI study, this progression concerned a rate of $16 \%$ per year during the first twelve months of the study (17) and previous studies defined a conversion between $10 \%$ and $17 \%$ per year (32), suggesting thus that our results were comparable and quite similar to the data from current cohorts.

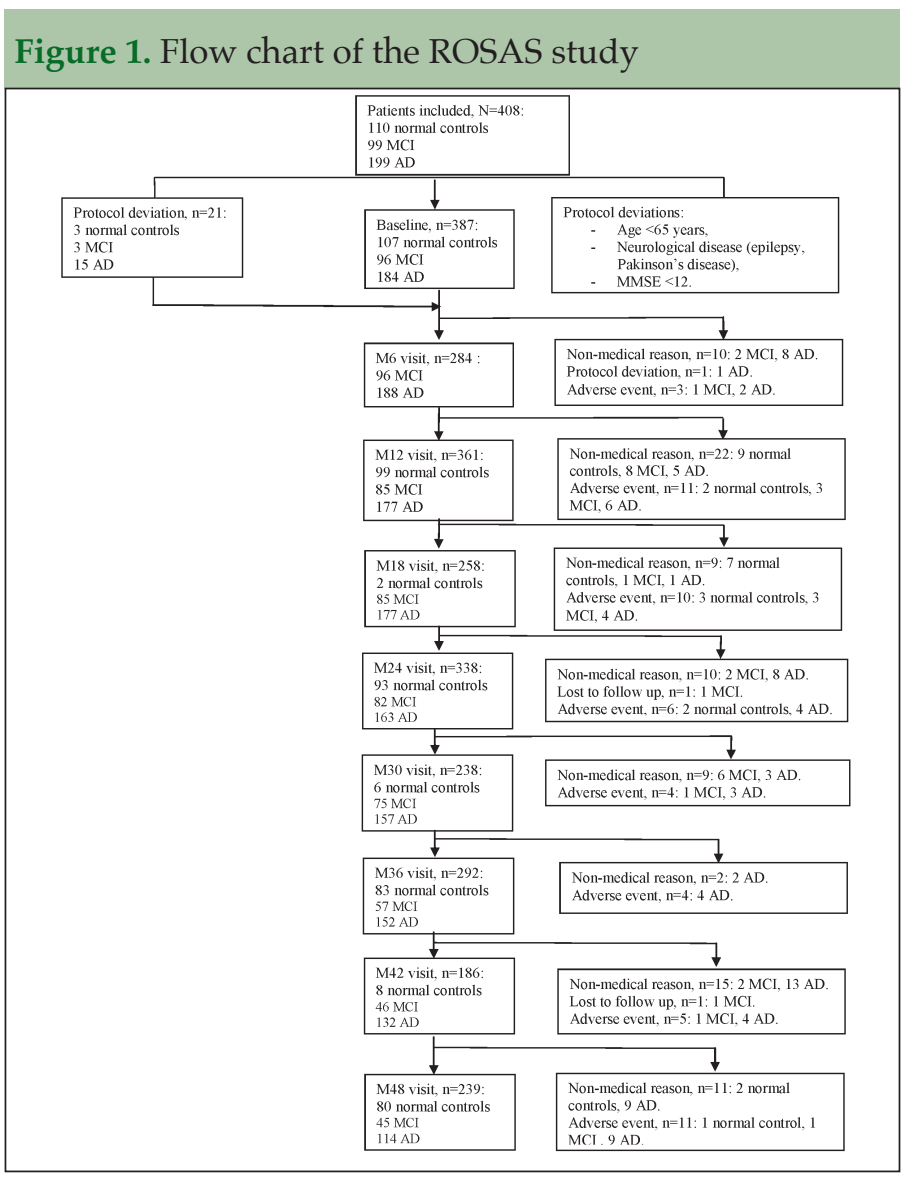

The ROSAS cohort is a unique and large French monocentric longitudinal prospective study that followed different patterns of cognitive evolution of MCI and AD subjects. To our knowledge, this study has been the largest monocentric cohort performed in the AD research and clinical memory center in France. Subsequently, the inclusion of participants in a highly-experienced memory center permitted homogeneity of the cohort and patients' evaluation, without a significant inter-center variation. In addition, this monocentric study was not only rich in clinical data collected but was improved by radiological data and biocollection. The ROSAS study provides a major opportunity, during a longer 4-year follow up, twice a year for participants with AD and MCI and once a year for subjects with normal neuropsychological tests, to describe and to analyze the evolution of decline and biomarkers in these three groups. Finally, the withdrawals for different reasons (medical reasons (deaths included) and non-medical reasons) represented 
$1 / 3$ of the cohort population. This result was lower than previously reported in other cohorts. In REAL.FR study (33), a French prospective multi centric cohort of $A D$ patients, $2 / 3$ of subjects did not complete a 4 -year follow up.

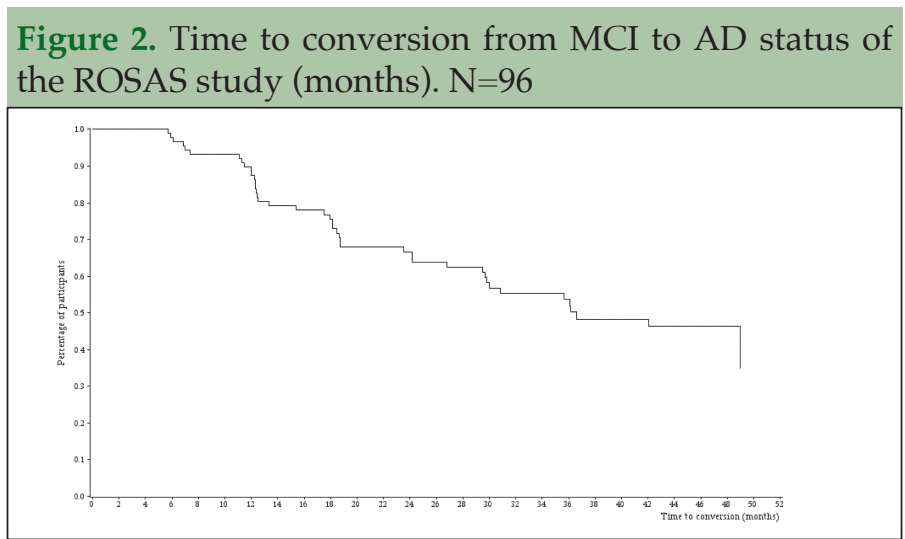

The ROSAS study had some limitations however. Firstly, the population recruited was not representative of $\mathrm{AD}$ and MCI subjects with "real populations". Secondly, the definition of the different groups (AD, MCI and control group) was based only on clinical criteria (DSMIV) since the lumbar puncture was only performed in few patients. Therefore, we did not have complete physio pathological biomarkers to confirm the diagnosis. However, the bio-collection from this cohort as well as neuroimaging data will be analyzed and should retrospectively improve the information on disease early phases and permit more precise diagnosis.

This current cohort will allow for additional analyses including imaging studies with brain MRI or protein biomarkers and will highlight important data on the earlier diagnosis. To date, the existing biochemical or neuroimaging biomarkers of $\mathrm{AD}$ have paved the way for an earlier diagnosis and a better understanding of the natural course of dementia. In the perspective of large population screening, ROSAS study focused mostly on blood or urinary samples that had been easier to collect and process - similar to current clinical practice in Europe. Multiple biomarkers have been used separately to quantify the disease progression. But, the identification of optional markers for predicting this process and explaining it for future therapies still remains unresolved. Nowadays, methods are also needed to integrate these multiple biomarkers to obtain a better recognition of the disease (34) predicting the emerging of AD with the ultimate goal of providing a platform for therapeutic intervention with disease modifying therapies (35).

Further analysis involving MRI, metabolomics analysis or clinical criteria (e.g. NPI) and results from the ROSAS study will help better characterization of the cognitive and behavioural course of the disease. Deeper analysis of factors differentiating MCI patients progressing to dementia from non -converters will be of particular value in addition to previous major studies $(28,32,35)$.
Funding: This study was supported by Institut De Recherche SERVIER - CL2NEURO-003 study protocol (registration number DGS 20060500).

Conflict of interest disclosure: Dr. Vera Kiyasova, Dr. Maria Pueyo, Mrs. Stéphanie Galtier, Mrs. Isabelle Guignot are employees of SERVIER Laboratories..;

Ethical standards: Ethics committee of Toulouse University Hospital approved the study protocol and all its amendments. The study was conducted in compliance with the protocol, GCP, the ethical principles that have their origin in the Declaration of Helsinki and the applicable regulatory requirements.

\section{References}

1. Winblad B, Amouyel P, Andrieu S, et al. Defeating Alzheimer's disease and other dementias: a priority for European science and society. Lancet Neurology. 2016;15(5):455-532.

2. Sperling RA, Aisen PS, Beckett LA, et al. Toward defining the preclinical stages of Alzheimer's disease: Recommendations from the National Institute on Aging-Alzheimer's Association workgroups on diagnostic guidelines for Alzheimer's disease. Alzheimer's \& Dementia: Journal of Alzheimer's Association. 2011;7(3):280-92.

3. Gaugler JE, Kane RL, Johnston JA, Sarsour K. Sensitivity and specificity of diagnostic accuracy in Alzheimer's disease: a synthesis of existing evidence. American Journal of Alzheimer's Disease and Other Dementias. 2013;28(4):337-47.

4. Jack CR, Holtzman DM. Biomarker Modeling of Alzheimer's Disease. Neuron. 2013;80(6):1347-58.

5. Blennow K, Hampel H, Weiner M, Zetterberg H. Cerebrospinal fluid and plasma biomarkers in Alzheimer disease. Nature Reviews Neurology. 2010;6(3):131-44.

6. Rowe CC, Villemagne VL. Brain amyloid imaging. Journal of Nuclear Medicine Technology. 2013;41(1):11-8.

7. Weimer DL, Sager MA. Early identification and treatment of Alzheimer's disease: Social and fiscal outcomes. Alzheimer's \& Dementia: Journal of Alzheimer's Association. 2009;5(3):215-26.

8. DSM-IV-TR. American Psychiatric Association; 2000. 982 p. (DSM Library). http://dsm.psychiatryonline.org/doi/abs/10.1176/appi. books.9780890420249.dsm-iv-tr

9. Folstein MF, Folstein SE, McHugh PR. « Mini-mental state ». A practical method for grading the cognitive state of patients for the clinician. Journal of Psychiatric Research. 1975;12(3):189-98.

10. Morris JC. The Clinical Dementia Rating (CDR): current version and scoring rules. Neurology. 1993;43(11):2412-4.

11. Rey A. L'examen clinique en psychologie. Presse Universitaire de France. Paris. 1964.

12. Román GC, Tatemichi TK, Erkinjuntti T, et al. Vascular dementia: diagnostic criteria for research studies. Report of the NINDS-AIREN International Workshop. Neurology. 1993;43(2):250-60.

13. Rosen WG, Mohs RC, Davis KL. A new rating scale for Alzheimer's disease. American Journal of Psychiatry. 1984;141(11):1356-64.

14. Reitan RM, Wolfson D. A selective and critical review of neuropsychological deficits and the frontal lobes. Neuropsychology Review. 1994;4(3):161-98.

15. Pedrosa H, De Sa A, Guerreiro M, et al. Functional evaluation distinguishes MCI patients from healthy elderly people--the ADCS/MCI/ADL scale. Journal of Nutrition Health and Aging. 2010;14(8):703-9.

16. Cummings JL, Mega M, Gray K, et al. The Neuropsychiatric Inventory: comprehensive assessment of psychopathology in dementia. Neurology. 1994;44(12):2308-14

17. Petersen RC, Aisen PS, Beckett LA, et al. Alzheimer's Disease Neuroimaging Initiative (ADNI). Neurology. 2010;74(3):201-9.

18. Sambrook R, Herrmann N, Hébert R, et al. Canadian Outcomes Study in Dementia: study methods and patient characteristics. Canadian Journal of Psychiatry. 2004;49(7):417-27.

19. Vellas B, Hausner L, Frölich L, et al. Progression of Alzheimer disease in Europe: data from the European ICTUS study. Current Alzheimer Research. 2012;9(8):902-12.

20. Barnes DE, Haight TJ, Mehta KM, et al. Secondhand smoke, vascular disease, and dementia incidence: findings from the cardiovascular health cognition study. American Journal of Epidemiology. 2010;171(3):292-302.

21. Kim G, Kim H, Kim KN, et al. Relationship of cognitive function with B vitamin status, homocysteine, and tissue factor pathway inhibitor in cognitively impaired elderly: a cross-sectional survey. Journal of Alzheimer's Disease. 2013;33(3):853-62.

22. Blasko I, Hinterberger M, Kemmler G, et al. Conversion from mild cognitive impairment to dementia: influence of folic acid and vitamin B12 use in the VITA cohort. Journal of Nutrition Health and Aging. 2012;16(8):687-94.

23. Faux NG, Ellis KA, Porter L, et al. Homocysteine, vitamin B12, and folic acid levels in Alzheimer's disease, mild cognitive impairment, and healthy elderly: baseline characteristics in subjects of the Australian Imaging Biomarker Lifestyle study. Journal of Alzheimer's Disease. 2011;27(4):909-22.

24. Moustafa AA, Hewedi DH, Eissa AM, et al. Homocysteine levels in 
Supplementary table 1. Main Neuropsychological endpoints at inclusion in the ApoE $\varepsilon 4$ carriers and non-carriers participants

\begin{tabular}{|c|c|c|c|c|c|c|c|}
\hline & \multicolumn{2}{|c|}{ Control Population } & \multicolumn{2}{|c|}{ MCI Population } & \multicolumn{2}{|c|}{ AD Population } \\
\hline & & $\begin{array}{c}\text { ApoE- } \\
(\mathrm{N}=85)\end{array}$ & $\begin{array}{c}\text { ApoE+ }+ \\
(N=22)\end{array}$ & $\begin{array}{c}\text { ApoE- } \\
(\mathrm{N}=62)\end{array}$ & $\begin{array}{c}\text { ApoE+ } \\
(\mathrm{N}=34)\end{array}$ & $\begin{array}{c}\text { ApoE- } \\
(\mathrm{N}=95)\end{array}$ & $\begin{array}{c}\text { ApoE+ } \\
(\mathrm{N}=88)\end{array}$ \\
\hline ADAS-COG 11 Total score & Mean \pm SD & NA & NA & $8.3 \pm 2.4$ & $8.6 \pm 2.7$ & $16.5 \pm 6.2$ & $18.4 \pm 8.9$ \\
\hline Global CDR score & Mean \pm SD & $0.01 \pm 0.05$ & $0.00 \pm 0.00$ & $0.48 \pm 0.09$ & $0.51 \pm 0.09$ & $1.01 \pm 0.45$ & $0.99 \pm 0.49$ \\
\hline
\end{tabular}

Supplementary table 2. Results of cerebrospinal fluid (CSF) samples analyses

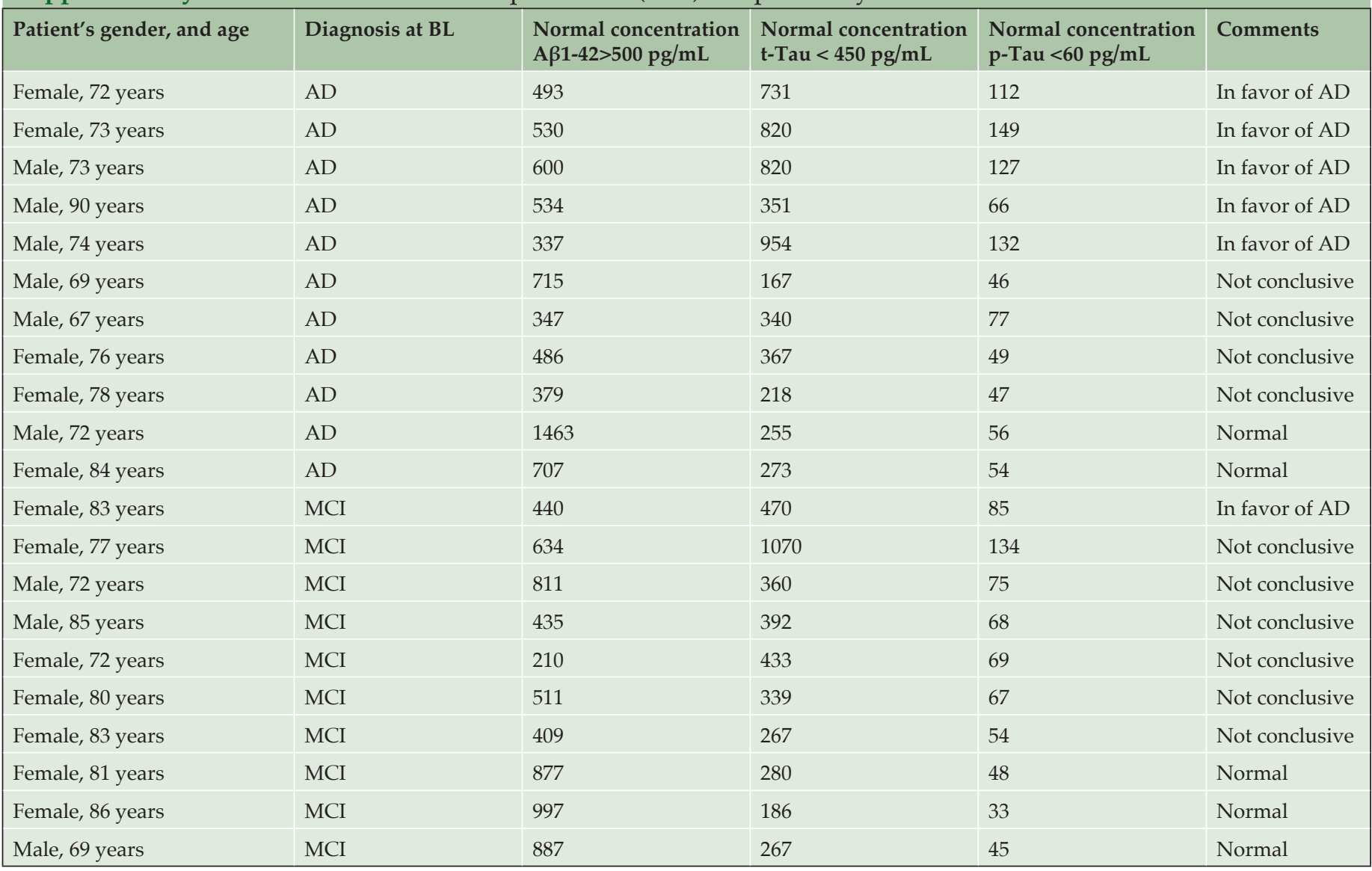

schizophrenia and affective disorders-focus on cognition. Frontiers in Behavioral Neuroscience. 2014;8:343.

25. Doody RS, Demirovic J, Ballantyne CM, et al. Lipoprotein-associated phospholipase A2, homocysteine, and Alzheimer's disease. Alzheimer's \& Dementia. 2015;1(4):464-71.

26. Rebeck GW, Reiter JS, Strickland DK, Hyman BT. Apolipoprotein E in sporadic Alzheimer's disease: allelic variation and receptor interactions. Neuron. 1993;11(4):575-80.

27. Holtzman DM, Bales KR, Tenkova T, et al. Apolipoprotein E isoformdependent amyloid deposition and neuritic degeneration in a mouse model of Alzheimer's disease. Proceedings of the National Academy of Sciences of the U S A. 2000;97(6):2892-7.

28. Ellis KA, Bush AI, Darby D, et al. The Australian Imaging, Biomarkers and Lifestyle (AIBL) study of aging: methodology and baseline characteristics of 1112 individuals recruited for a longitudinal study of Alzheimer's disease. International Psychogeriatrics Association. 2009;21(4):672-87.

29. Ellis KA, Szoeke C, Bush AI, et al. Rates of diagnostic transition and cognitive change at 18-month follow-up among 1,112 participants in the Australian Imaging, Biomarkers and Lifestyle Flagship Study of Ageing (AIBL). International Psychogeriatrics. 2014;26(4):543-54.
30. Schneider LS, Insel PS, Weiner MW. Treatment With Cholinesterase Inhibitors and Memantine of Patients in the Alzheimer's Disease Neuroimaging Initiative. Archives of Neurology. 2011;68(1):58-66.

31. Reynish E, Cortes F, Andrieu S, Cantet C, et al. The ICTUS Study: A Prospective longitudinal observational study of 1,380 AD patients in Europe. Study design and baseline characteristics of the cohort. Neuroepidemiology. 2007;29(1-2):29-38.

32. Landau SM, Harvey D, Madison CM, et al. Comparing predictors of conversion and decline in mild cognitive impairment. Neurology. 2010;75(3):230-8.

33. Gillette-Guyonnet S, Nourhashemi F, Andrieu S, et al. The REAL.FR research program on Alzheimer's disease and its management: methods and preliminary results. Journal of Nutrition Health and Aging. 2003;7(2):91-6.

34. Kohannim O, Hua X, Hibar DP, et al. Boosting power for clinical trials using classifiers based on multiple biomarkers. Neurobiology of Aging. 2010;31(8):1429-42.

35. Petersen RC. Prediction and Prevention of Alzheimer's Disease. Lancet Neurology. 2010;9(1):4-5. 